\title{
UNIVERSITYOF
}

FORWARD

THINKING

WESTMINSTER用

WestminsterResearch

http://www.westminster.ac.uk/westminsterresearch

Using micro-blogging to dismantle knowledge hierarchies in product development teams

Evans R.D. and Rafique M.

This is a copy of the author's accepted version of Evans R.D. and Rafique M. (2015) Using micro-blogging to dismantle knowledge hierarchies in product development teams, subsequently published in The Proceedings of the IEEE International Conference on Knowledge-Based Engineering and Innovation Tehran, Iran 05 Nov 2015 IEEE .

It is available online at:

https://dx.doi.org/10.1109/KBEl.2015.7436025

(C) 2015 IEEE . Personal use of this material is permitted. Permission from IEEE must be obtained for all other uses, in any current or future media, including reprinting/republishing this material for advertising or promotional purposes, creating new collective works, for resale or redistribution to servers or lists, or reuse of any copyrighted component of this work in other works.

The WestminsterResearch online digital archive at the University of Westminster aims to make the research output of the University available to a wider audience. Copyright and Moral Rights remain with the authors and/or copyright owners.

Whilst further distribution of specific materials from within this archive is forbidden, you may freely distribute the URL of WestminsterResearch: ((http://westminsterresearch.wmin.ac.uk/)).

In case of abuse or copyright appearing without permission e-mail repository@westminster.ac.uk 


\section{Using micro-blogging to dismantle knowledge hierarchies in product development teams}

\author{
Richard David Evans \\ Business Information Management and Operations \\ University of Westminster \\ London, United Kingdom \\ R.Evans@westminster.ac.uk
}

\author{
Muhammad Rafique \\ Engineering Management \\ National University of Science and Technology \\ Islamabad, Pakistan \\ raf.sibi@ceme.nust.edu.pk
}

\begin{abstract}
The traditional assumption by academics and industrialists that 'Experts' are more valuable to an organisation than inexperienced workers is one which should be challenged. Typically, those considered experts employ tried and tested methods for production success passed on by elders with wisdom, while inexperienced knowledge workers often remain unheard and their knowledge under-utilised. However, less experienced workers with an openness to creativity and fresh perspectives to engineering practice might possess the knowledge required to innovate manufacturing projects and sustain competitive advantage. This article extends the research of Tempest [1] and Evans et al. [2] by challenging the unilateral transfer of knowledge seen in traditional product development teams. A methodological framework is presented aimed at dismantling knowledge hierarchies by creating an 'Anonymous Worker' environment using micro-blogging which can be embedded into production departments. Potential limitations to the successful implementation of the framework are reported, identifying that the framework would be most beneficial applied to the start of a product's lifecycle during the conceptualisation of ideas phase.
\end{abstract}

Keywords-Corporate Learning; Knowledge Hierarchies; Manufacturing Knowledge Sharing; Micro-Blogging; Product Development Teams.

\section{INTRODUCTION}

Since the 1990s and given the ever more challenging economic conditions experienced by companies today, there has been increasing recognition that one of the most valuable resources owned by organisations is employee knowledge. Prior to the 1990s, Porter and Millar [3] suggested that the key to companies being successful was in the information they possess. Nowadays, it is believed that knowledge is the key to corporate survival [4]. Nonaka [5] predicted that successful companies will be those that create, capture and make best use of their knowledge and then apply it to new innovative Product Development (PD). The need to manage and develop this knowledge effectively has increasingly been acknowledged in order that organisations may respond to the challenges presented in today's dynamic and complex business environment and to overcome economic uncertainties [6]. In the last two decades, this challenge has grown considerably with the development of the Worldwide Web (WWW) and social media sites, such as Facebook.com and Twitter.com. Companies are now faced with many opportunities, but also many problems and challenges which in-turn creates a need for refinement of the traditional knowledge hierarchy.

In today's competitive global market place, manufacturing companies, in particular, have needed to develop flexible and responsive work processes to ensure competitive advantage [7]. The response from industry has seen an increased focus on lean, green and agile practices, which aim to: eliminate waste and the use of substances harmful to the environment; reduce product development and manufacturing time and cost; improve quality and collaboration; and ideally, "get it right first time" [8]. As organisations strive to maintain and develop their competitive edge, successful PD and the ability to introduce innovative new product platforms quickly have been identified as fundamental to corporate success [9]. It is no longer sufficient to simply re-engineer existing product offerings if organisations wish to survive and thrive. Especially in relation to the engineering and high technology sectors, effective Knowledge Management (KM) is paramount [10]. Failure to achieve this can ultimately restrict employee access to key information, fail to address product defects and reduce opportunities for innovation. The creation of a corporate culture which allows its employees to share their experiences, has become a crucial goal for manufacturing organisations. Corporate leaders are now seeking methods to capture, manage and share their employees' knowledge around the business.

One method identified by Tempest [1] is to create an open, inclusive and fun socio-psychological working environment where employees collaboratively work together, openly criticizing and debating with one another to help solve problems and identify innovative enhancements to new and existing products, thus assisting with the dismantling of 


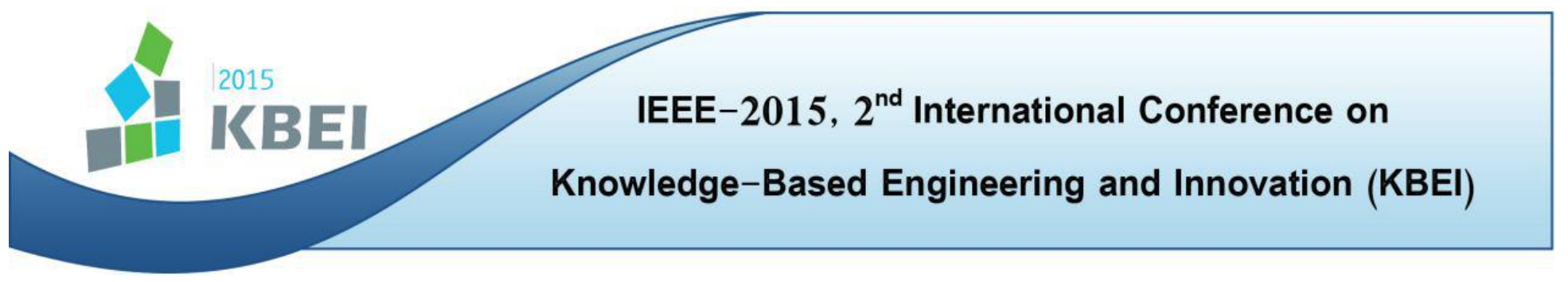

knowledge silos and traditional knowledge hierarchies which have emerged in modern day team working.

This article extends the research of both Tempest [1] and Evans et al. [2] by exploring the role of micro-blogging in challenging the unilateral transfer of knowledge typically observed in PD teams from less experienced employees (Novices) to Subject Matter Experts (SMEs). The term 'expert' signifies a person who is more knowledgeable than a standard employee and thus seen as more valuable to the organisation. However, this assumption should be challenged due to changing market conditions brought about by the WWW. Research relating to $\mathrm{KM}$ has historically reported on knowledge transfer being from an expert to a novice, or person with limited expertise [11, 12]; this is best observed in the traditional 'apprenticeship model', shown in Fig. 1, which sees an 'expert or 'master' pass on their acquired knowledge to a typically young apprentice beginning their career'.

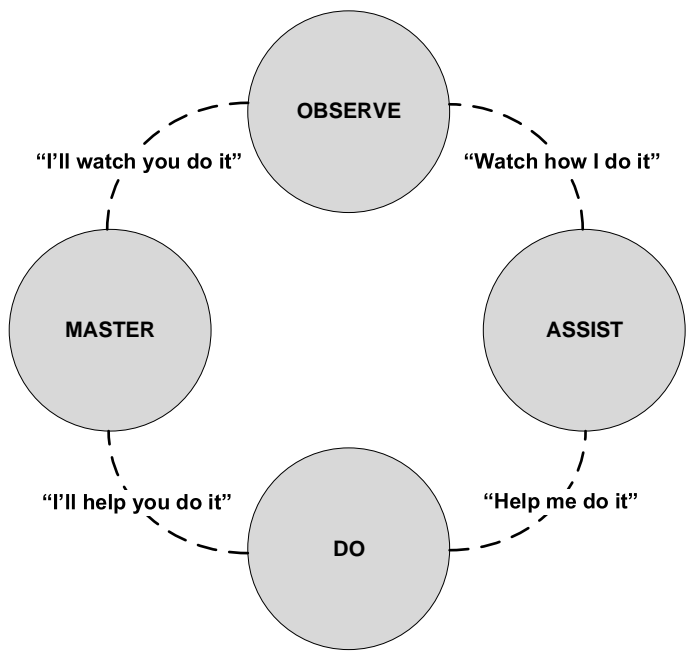

Fig. 1. Traditional Apprenticeship Model

However, many researchers $[1,11,13]$ dispute this norm, stating that knowledge should be captured from diverse members representing different categories of the knowledge hierarchy and that companies may benefit from this broad knowledge base especially if dealing in uncertain marketplaces. One example of successfully dismantling this hierarchical structure is seen in Silicon Valley, California, where high-tech organisations are more often recruiting younger employees due to their experience and greater exposure to developing WWW technologies. For example, the median age of an employee at Google is 29, at Facebook it is 28 and at Zynga it is 26. In fact, many CEOs of start-ups and some established companies in the 'Bay Area' are under 30 (Quirky (26), CodeForce 360 (27), Rally.org (30)), with analysts stating that there is now ageism towards the older generation of employee. Office structures are also changing, with pool and table tennis tables in the office environment not being uncommon.

\section{THE KNOWLEDGE HIERARCHY}

The development of new and existing products traditionally embraces corporate strategy, market research and analysis, the development and testing of prototypes and production planning. Nowadays, however, this has been extended to include a wider range of corporate activities which now has to accommodate the geographical dispersement of product development teams. This creates a need for web-based solutions which aid employee collaboration and knowledge sharing across physical boundaries.

The management of products during their lifecycle progress through several stages, from product conceptualisation, design and manufacture to service and disposal, as illustrated in Fig. 2.

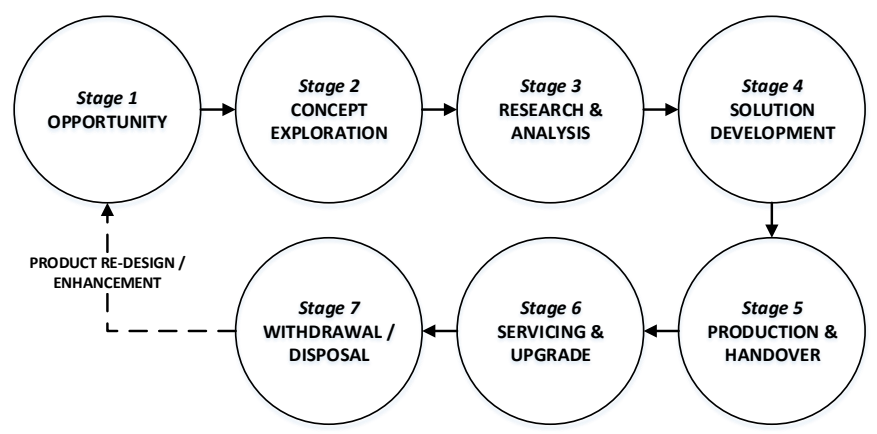

Fig. 2. Generic Product Development Process

Within product development teams, employees rise through a defined hierarchy of knowledge until they achieve either Chief Product Officer status or become vice president of PD. This hierarchy creates an inter-generational divide from novice to expert where the knowledge of standard employees rising up the hierarchy, as is shown in Fig. 3, may be overlooked by managers residing in a more senior hierarchy. Newly appointed employees who have recently graduated from University, been redeployed or moved from another company may offer solutions or ideas which had not been considered previously and may remain uncaptured or ignored until they reach a certain level in the hierarchy. This conundrum suggests a more inclusive team environment should be created to incorporate newly acquired knowledge into the PD process

SMEs, whom typically hold a senior role, develop their expertise through the learning of knowledge transferred by elders through seniority who developed wisdom over time of successful production practices. However, this 'hand-medown' of wisdom may lead to overlooked knowledge or the undermining of knowledge from certain groups of workers in the hierarchy. Eventually, this wisdom, added only to by senior 


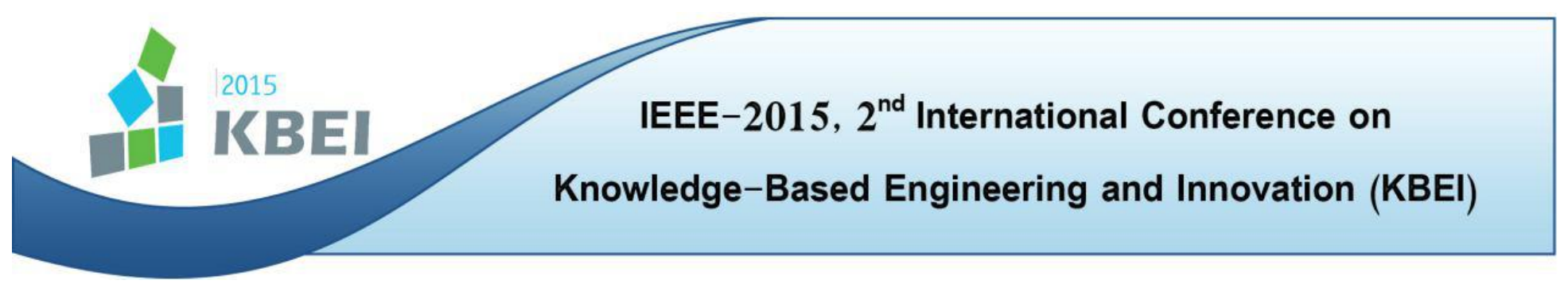

employees, will become institutionalized and be embedded into standard working practices. This practice may ultimately lead to innovation only occurring in the 'Competent - Expert' brackets of the hierarchy, with less experienced employees not being heard and eventually becoming frustrated. This could lead to the alienation of knowledgeable employees which have yet to reach the higher levels of the hierarchy where their ideas and developing knowledge are heard and taken seriously.

A key opportunity emerges, therefore, to combine intergenerational knowledge through the use of social media tools. By creating a micro-blogging environment which assigns employees generic usernames and profile images, disregarding their position in the hierarchy, organisations may amalgamate the knowledge of experienced workers with the knowledge of 'next generation' young engineers who possess fresh perspectives to current engineering practice. Tempest [1] adds that by creating this type of environment which disregards age, hierarchical position and experience, a state of 'shared learning' may be developed which can result in the "mixing of enthusiastic, but less experienced people, with more experienced individuals who understand the practical issues of the realization of ideas and project feasibility". Of course, other methods do exist which allow employees to disseminate their knowledge to the wider workforce, including job rotations, secondments to inter-linked departments and international postings, which allow employees to experience different cultures and working practices. However, micro-blogging is one method which still remains relatively un-explored.

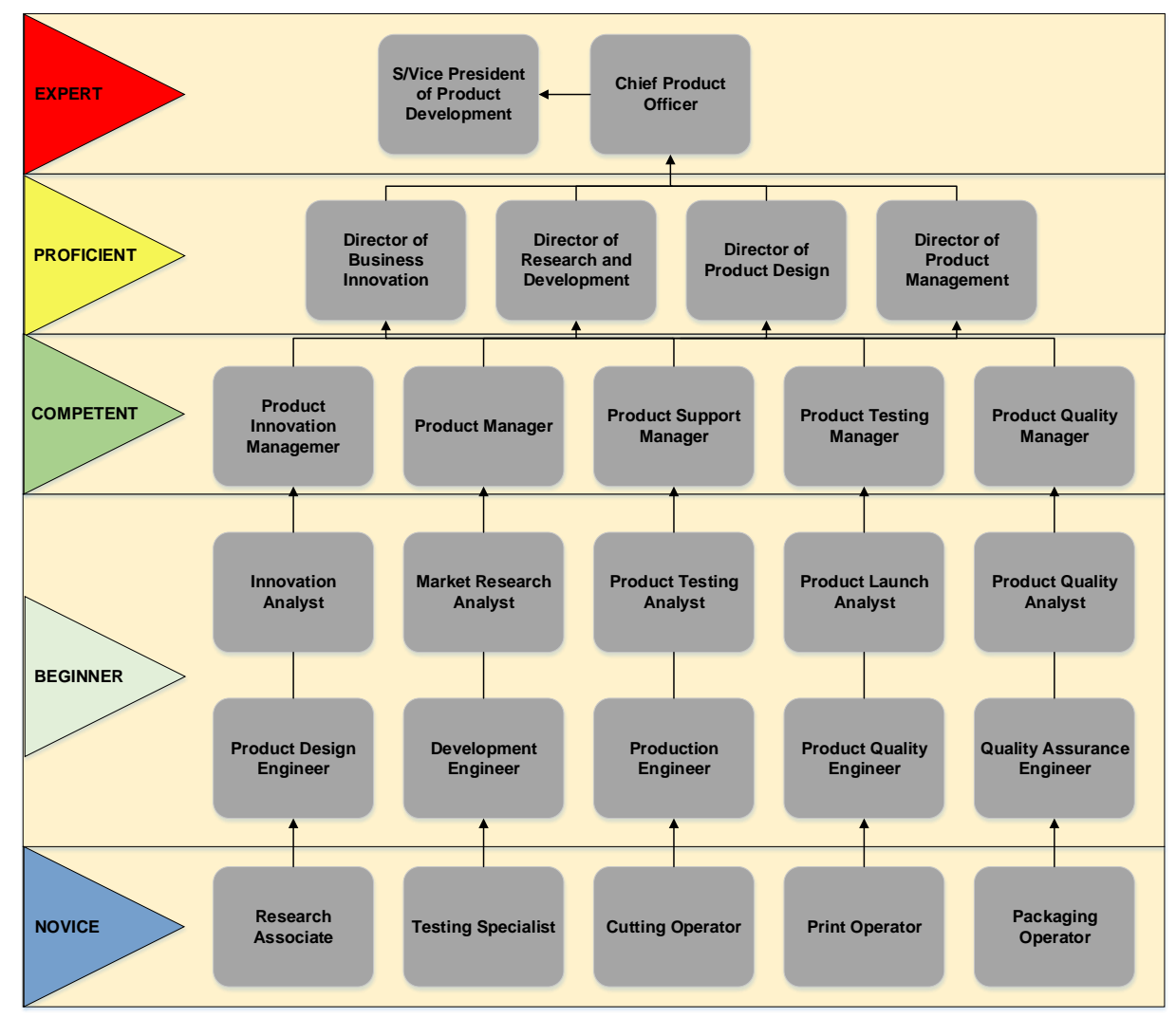

Fig. 3. Generic Knowledge Hierarchy in Product Development Teams

Product development teams are seen as knowledgeintegrating mechanisms. However, experts typically follow the status quo from product conceptualisation to project completion, storing and re-using historical information for decision making and not often seeking new knowledge from less experienced employees. By ignoring this new knowledge, organisations fail to capture potentially innovative ideas to manufacturing problems. By creating a more open and inclusive working environment where workers are not categorised against a knowledge hierarchy may facilitate 


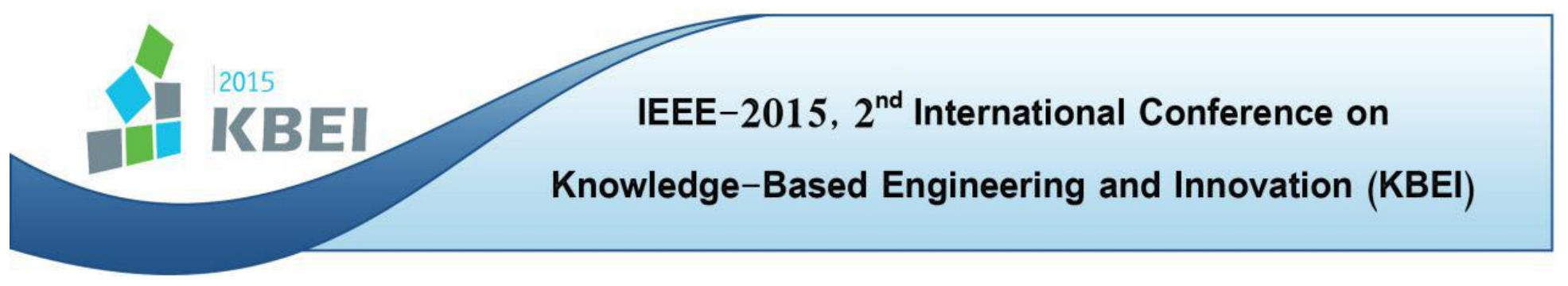

improved knowledge sharing amongst teams, where views are considered and heard by senior employees whom have decision making power [14]. However, knowledge must be extracted from employees and incentives must be put in place in exchange for valuable knowledge contribution; this, however, is out of scope of this research. Von Krogh and Roos [13] state that new knowledge is often acquired by trial and testing and, therefore, is more than the mere transfer of knowledge from an expert to a novice. Knowledge sharing is a social process [15] which requires different perspectives from diverse employee groups within an organisation [14, 16]; a blended approach should be taken incorporating workers at different levels of the hierarchy into the process, thus enabling improved learning and innovation [1]. It is for this reason that micro-blogging should be explored further and could potentially create the right opportunity for a more open, fun and inclusive knowledge sharing environment. By employing micro-blogging for the process of knowledge management, organisations are able to connect people to people and people to information more effectively; facilitate connectivity, sharing and collaboration across boundaries; capture a wide base of typically informal or highly dispersed views and information; help colleagues identify and locate previously unknown experts; and Help employees discover organisational knowledge $[17,18,19]$.

\section{Proposed CONCEPTUAL FrameWORK}

Based on the logic that knowledge sharing is a social process which should encapsulate different categories of worker from the knowledge hierarchy shown in Fig. 3, we have designed a methodological framework, shown in Fig. 4, which builds upon the work of Evans et al. [2]. The process demonstrates how different categories of knowledge worker can remain anonymous by using generic micro-blogging handles and profile images which are automatically assigned to them at the sign-up stage of micro-blogging site.

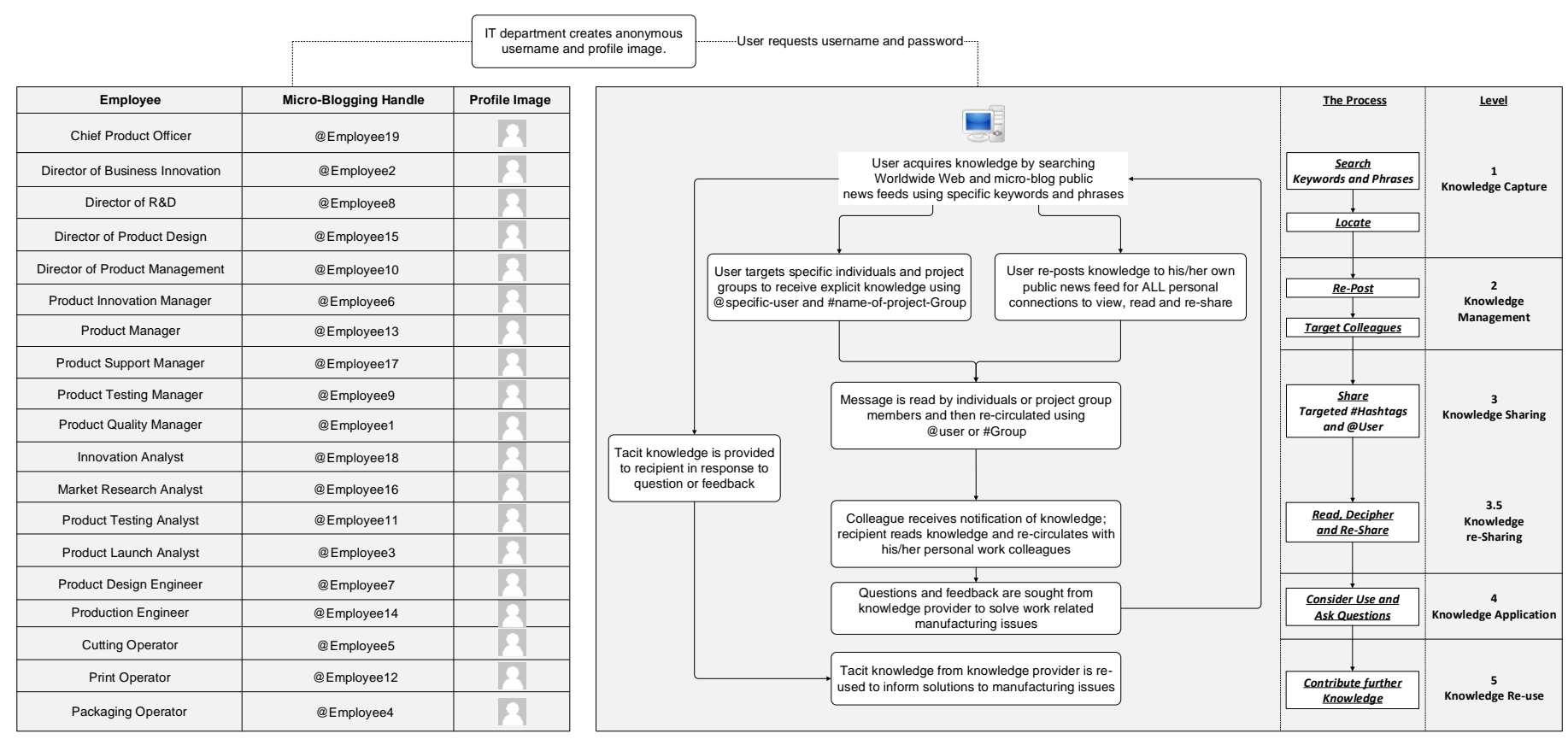

Fig. 4. Framework for Dismantling Knowledge Hierarchies

The framework places an employee at the centre of its process, not defined by the hierarchy. This enables any employee in the hierarchy to contribute knowledge they have accumulated, regardless of their stature in ranking, which can be shared with all employees, including those senior in rank. Workers from different levels of the hierarchy can contribute collectively to the 'team knowledge base', rather than the accumulation of knowledge by knowledge-rich employees at the top of the hierarchy being passed on to the knowledge-poor at the lower levels of the hierarchy. For this reason, the proposed framework issues all employees an 'anonymous handle' which should allow for greater knowledge composition i.e. the combined knowledge of all team members. By creating an anonymous environment where users all sit horizontally in one knowledge hierarchy and are unaware to whom they are communicating with, the traditional hierarchy becomes 


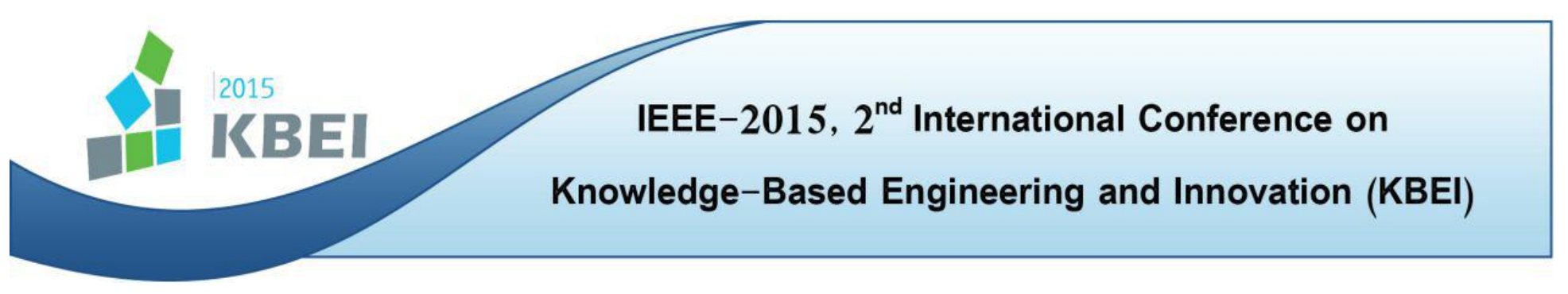

somewhat dismantled with users not knowing if the person with which they are speaking is above or below them in the hierarchy; this enables a more inclusive and fun environment where all employee knowledge may be acknowledged as good or bad without fear of repercussion. Of course, barriers to this environment being successful do exist; these are discussed in section 4 .

Finally, March [20] suggested that knowledge creation has two forms: Exploitative and Explorative. The proposed framework combines both forms, allowing experienced workers to use existing knowledge and less experienced workers to introduce new assumptions and techniques. It is hypothesized, but out of scope of this article, that by creating an anonymous web-based environment where colleagues are unknown to one another, should create a team culture where criticism is tolerated and debates are frequent, thus enabling all categories in the knowledge hierarchy to contribute innovatively to product development projects.

\section{A. Knowledge Contribution Voting Mechanism}

In order to determine knowledge-worthy contributions, it is proposed that a voting mechanism is embedded into the microblogging framework, as shown in Fig. 5. By adding this mechanism, those with decision making power are able to view which knowledge contributions are considered good or bad ideas by colleagues, thus creating a user-generated feedback mechanism.

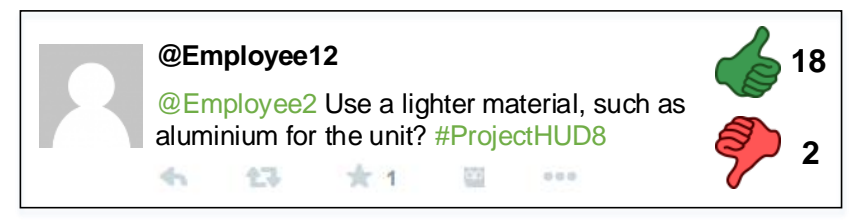

Fig. 5. Generic Product Development Process

As shown, users contribute their knowledge which is then voted as either 'good' / green thumbs-up or 'bad' / red thumbsdown by anonymous colleagues. Decision makers may then have this information fed back to them via their user account or by viewing each contribution individually.

\section{POTENTIAL IMPLICATIONS}

It is recognised [21] that the activities associated to the capture, management and sharing of employee knowledge face numerous barriers typically relating to either social factors or the technology adopted or a combination of both [22]. Disterer [23] and Riege [24] both recognised that organisations themselves can contribute to a failure to collect knowledge, stating that the hierarchical structure of a company may be counter-productive to knowledge capturing initiatives. Disterer
[23] and Ardichvili [21] added that individuals often perceive knowledge as power that can allow them to advance within an organisation and, therefore, are not prepared to share. Riege [24] further added that individuals often do not have the time to contribute knowledge to others, if they are working, for example, on busy production or assembly lines.

Various barriers to KM may be identified in relation to the technologies which assist in the capture and distribution of knowledge. Riege [24] commented on the interoperability problems associated with the introduction of new knowledge management systems into existing IT infrastructures. Compatibility issues with current or legacy systems may also arise when trying to extract previously published knowledge. With regard to micro-blogging, barriers to adoption may occur. From an accessibility standpoint, all users must have open and un-restricted access to the site from their desktop computer and portable devices to allow them to contribute ideas and suggestions whilst outside of the workplace. Managers must provide sufficient time for contributing to and searching existing knowledge; however, this could cause social 'chat' to take place which would require monitoring. Limitations on the number of characters per micro-blog message may also cause a hindrance and may cause some users to become demotivated to use the technology.

Trust is a key issue. Employees within organisations often will not share their tacit knowledge for fear that other individuals will take credit for the knowledge they have previously shared [24]. From an organisational point of view, many companies do not share knowledge with external partners for fear of divulgence of confidential information, including pricing schedules and customer databases. Thomas [25] pointed out that some organisations do not offer sufficient training in connection with the introduction of new systems, causing difficulties and misunderstanding to many employees being unfamiliar with the new system installed. Additionally, companies often do not explain the benefits of new IT systems to its employees and, consequently, users are not naturally inclined to use them. In addition, a lack of consistent policies across businesses often hinders the successful management of information systems.

Finally, a practical implication must be noted for those adopting the proposed framework. Through informal face-toface meetings with 15 members of a global manufacturing organisation, it is believed that the framework would be best utilised during the product conceptualisation phase of the product lifecycle. This is due to the need for innovative ideas to be delivered during this phase, when brainstorming sessions are held and market research is conducted. 


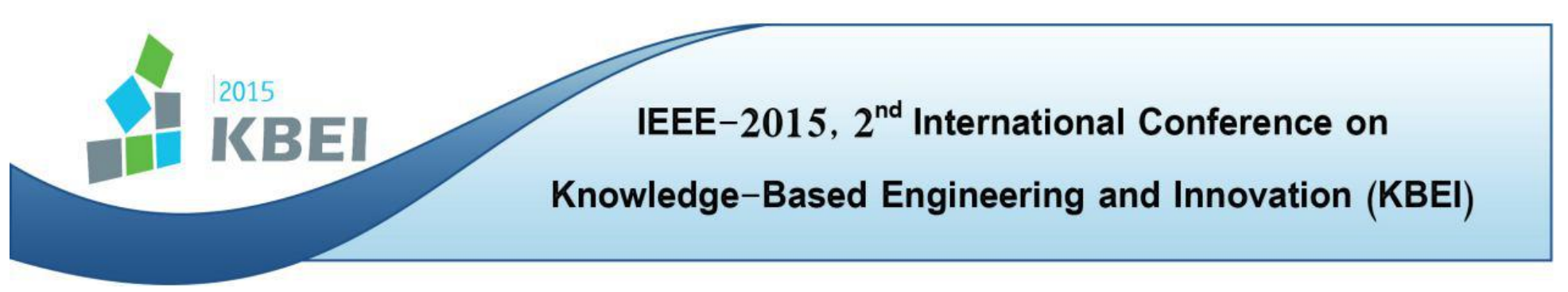

\section{CONCLUSION}

This research has attempted to add to existing research on knowledge hierarchies by aiming to identify the role microblogging can play in dismantling hierarchies found in traditional PD teams. We propose a conceptual framework which creates an anonymous web-based environment where all users are placed in a horizontal hierarchy and assigned generic usernames and profile images. In order to determine the credibility and success of the proposed framework, a validation study should be conducted within a real-world product development setting to identify its benefits and drawbacks. This is planned between Q4 2015 and Q1 2016; results of this study will be reported on in a subsequent article. We have established that micro-blogging can help organisations improve employee knowledge sharing. However, limitations do exist. The introduction of micro-blogging can provide employees with real-time access to knowledge and allow them to raise questions with knowledge producers outside of their hierarchy category. Micro-blogging facilitates and encourages communication through less formal and potentially more inclusive channels, with individuals employing established communication practices used in their social lives. In future, research must explore barriers which impede the dismantling of knowledge hierarchies and what repercussions may be present if hierarchies were to become more horizontal. Finally, the performance of employees when using such social media tools in the workplace must be scrutinized to analyse productivity output in using social media in organisations.

\section{REFERENCES}

[1] S. Tempest, "Intergenerational Learning: A Reciprocal Knowledge Development Process that Challenges the Language of Learning," Management Learning, vol. 34, pp. 181-200, 2003.

[2] R. Evans, J. Gao, O. Owodunni, S. Shah, S. Mahdikhah, M. Messaadia and D. Baudry, "A Framework for Improving the Sharing of Manufacturing Knowledge through Micro-Blogging," in Advances in Production Management Systems. Innovative and Knowledge-Based Production Management in a Global-Local World, 2014, pp. 19-26.

[3] M.E. Porter and V.E. Millar, "How Information Gives You Competitive Advantage," Harvard Business Review, vol. 63, pp. 149-160, 1985.

[4] S. Tseng, "A Study on Customer, Supplier and Competitor Knowledge Using the Knowledge Chain Model," International Journal of Information Management, vol. 29, pp. 488-496, 2009.

[5] I. Nonaka, "The Knowledge-Creating Company," Harvard Business Review, vol. 69, pp. 96-104, 1991.

[6] C. Rebolledo and J. Nollet, "Learning from suppliers in the aerospace industry," International Journal on Production Economics, vol. 129, pp. 328-337, 2011.

[7] A. Gunasekaran, E. Tirtiroglu and V. Wolstencroft, "An investigation into the application of agile manufacturing in an aerospace company," Technovation, vol. 22, pp. 405-415, 2002.

[8] V. Crute, Y. Ward, S. Brown and A. Graves, "Implementing Lean in aerospace - challenging the assumptions and understanding the challenges," Technovation, vol. 23, pp. 917-928, 2003.
[9] S. Ottosson, "Dynamic product development - DPD," Technovation, vol. 24, pp. 207-217, 2004.

[10] S. Liu and R.I.M. Young, "Utilising information and knowledge models to support global manufacturing co-ordination decisions," International Journal of Computer Integrated Manufacturing, vol. 17, pp. 479-492, 2004.

[11] T.H. Davenport and L. Prusak, Working Knowledge, Boston, USA, Harvard Business School Press, 1998.

[12] T.A. Stewart, Intellectual Capital: The New Wealth of Organizations, New York, USA, Bantam Books.

[13] G. Von Krogh and J. Roos, "Conversation Management," European Management Journal, vol. 13, pp. 390-394, 1995.

[14] J. Coopey and J. Burgoyne, "Politics and Organizational Learning," Journal of Management Studies, vol. 37, pp. 869-885, 2000.

[15] I. Nonaka and H. Takeuchi, The Knowledge-Creating Company, New York, USA, Oxford University Press, 1995.

[16] M. Easterby-Smith, M. Crossan and D. Nicolini, "Organisational Learning: Debates Past, Present and Future," Journal of Management Studies, vol. 37, pp. 783-796, 2000.

[17] R.D. Evans, J.X. Gao, N. Martin and C. Simmonds, "An Investigation into the Potential Use of Social Media Technologies to Improve the Product Development Functions within the Aerospace and Defence Industry" in International Conference on Manufacturing Research, Birmingham, UK, 2012, pp. 718-723.

[18] R.D. Evans, J.X. Gao, N. Martin and C. Simmonds, "Using Web 2.0Based Groupware to facilitate Collaborative Design in Engineering Education Scheme Projects" in International Conference on Interactive Collaborative Learning, Dubai, United Arab Emirates, 2014, pp. 397402.

[19] R.D. Evans, J.X. Gao, S. Mahdikhah, M. Messaadia and D. Baudry, "A Review of Crowdsourcing Literature Related to the Manufacturing Industry," Journal of Advanced Management Science, vol. 4, pp. 224$321,2016$.

[20] J.G. March, "Exploration and exploitation in organizational learning," Organization Science, vol. 2, pp. 71-87, 1991.

[21] A. Ardichvili, M. Maurer, W. Li, T. Wentling and R. Stuedemann, "Cultural influences on knowledge sharing through online communities of practice," Journal of Knowledge Management, vol. 10, pp. 94-107, 2006.

[22] D.J. Bradfield, "A Prototype Method and Tool to Facilitate Knowledge Sharing in the New Product Development Process," Ph.D. dissertation, Applied Sciences, Cranfield University, 2007.

[23] G. Disterer, "Individual and Social Barriers to Knowledge Transfer" in Hawaii International Conference on System Sciences, Hawaii, USA, 2001.

[24] A. Riege, "Three-dozen knowledge-sharing barriers managers must consider," Journal of Knowledge Management, vol. 9, pp. 18-35, 2005.

[25] E. Thomas, "Supplier integration in new product development: Computer mediated communication, knowledge exchange and buyer performance," Journal of Industrial Marketing Management, vol. 42, pp. 890-899, 2013. 